\title{
Variations of the flanker paradigm: Assessing selective attention in young children
}

\author{
Jennifer Martin McDermott, Koraly Pérez-Edgar, and Nathan A. Fox \\ University of Maryland, College Park, Maryland
}

\begin{abstract}
The development of selective attention and associated self-regulatory processes was assessed in young children, ages 4,5 , and 6 , through the use of three alternative versions of the flanker paradigm utilizing colors, shapes, and fish. These variations were used to examine the influence of task differences on children's performance. The presence of cognitive self-regulatory strategies in young children was also assessed. Significant flanker interference effects, marked by significant task-linked response time differences, were found across all three versions of the paradigm. Although a significant portion of children demonstrated self-regulatory abilities, not every participant demonstrated the specific strategies of self-monitoring and response control. Furthermore, these differences were evident across all age groups. The implications of these results are discussed within the theoretical context of task development, taking into consideration the need to modify computerized attention paradigms for use with young children in order to reliably measure cognitive constructs across children and adults.
\end{abstract}

Research has long suggested developmental differences in the ability to effectively translate incoming information into appropriate responses, particularly in the presence of interfering stimuli. Referred to as "selective attention," this ability requires an individual to focus attention on a target stimulus while simultaneously filtering out competing information from irrelevant stimuli. Previous studies on selective attention have indicated that filtering processes are different in children than in adults. Specifically, younger subjects are less able to adapt to variations in spatial configurations, less efficient at focusing attention on a target location, and also less likely to correctly inhibit the processing of irrelevant task stimuli (Enns \& Akhtar, 1989; Enns \& Cameron, 1987; Enns \& Girgus, 1985). Yet, there are several instances of children demonstrating intact cognitive skills typically attributed only to older children and adults, especially when standard task parameters were manipulated (Enns \& Akhtar, 1989; Müller, Zelazo, Hood, Leone, \& Rohrer, 2004; Pritchard $\&$ Neumann, 2004). These mixed results emphasize the importance of considering task design, including stimuli choice and protocol, when attempting to assess developmental differences in attention processes.

Furthermore, when investigating cognitive processes that change across the life span, it can be particularly difficult to construct a clear-cut developmental picture due to a shortage of testing measures that accurately elicit the same underlying skills in children and adults. Children have far less prior knowledge to bring to a testing situation than do adults. Consequently, performance deficits in children may not be due to developmental differences in the construct under investigation but instead may re- sult from inappropriate task requirements that exceed a child's experience range or that lead to children's misinterpretation of task instructions (Tipper \& McLaren, 1990; Wright, Waterman, Prescott, \& Murdoch-Eaton, 2003). According to Carver, Livesey, and Charles (2001, p. 47), "in order to make unambiguous cross-age comparisons it is necessary to find a single measure which is appropriate for a wide variety of ages and developmental stages."

Despite considerable work over the span of decades (Lane \& Pearson, 1982), the typical developmental trajectory for the mechanisms underlying attentional processes remains unclear. Early theoretical accounts have posited that age-related differences in cognitive performance can be attributed to the malleable composition of cognitive capacity (Halford, 1993). Capacity has been viewed as consisting of both storage and information processing levels, each of which can be drawn upon to various degrees during cognitive tasks. With age, children's information processing efficiency should increase and thus lead to increased room for storage or additional information processing. From this perspective, total capacity remains fairly stable, but the manner in which it is utilized changes with development (Case, 1985; van der Molen, 2000).

A relatively more recent notion argues that developmental changes in cognitive skills are more heavily dependent on inhibitory control mechanisms. Building on the notion of cognitive capacity, Bjorklund and Harnishfeger (1990) proposed that the ability to control inhibition leads to more selective intake of information and thereby increases the capacity available for more effective information processing. In contrast, Dempster (1993) has proposed that inhibition emerges as a by-product of brain maturation in the

J. M. McDermott, jnmartin@umd.edu 
frontal lobes. It becomes difficult to distinguish between these models when one is attempting to assign specific operational definitions to the concepts of inhibition and interference, since the terminology of active suppression and competitive interaction (Harnishfeger, 1995) can be applied to various processes in both of the previously mentioned models (see van der Molen, 2000, for a review).

Other theories have highlighted a central, overarching structure believed to guide actions and control lower-order mechanisms of attention processes. These models focus on supervisory systems (Shallice, 1988) and top-down processing as the directors of inhibition (Desimone \& Duncan, 1995), emphasizing both the suppression of distractors and the overriding of competitive information as primary mechanisms leading to conflict reduction and the implementation of cognitive control. Along this line, Casey and colleagues (Casey, Tottenham, \& Fossella, 2002) have investigated the neural circuitry implicated in cognitive control and found strong evidence that the basal ganglia are involved with the inhibition of competing responses, whereas the frontal cortex functions to maintain relevant information over time.

Although great progress has been made in identifying specific brain regions associated with various cognitive skills such as inhibition, much remains to be learned about the development of inhibition mechanisms in both typical and atypical populations. Overall, we know that the ability to inhibit is central to a broad range of tasks and serves an important function in information processing and response execution. As children age, they display more control over prepotent responding, as well as less vulnerability to distracting stimuli (Burack \& Enns, 1997; Diamond, 1990; Müller et al., 2004; Ridderinkhof \& van der Molen, 1995). These changes have been attributed to the emergence of inhibition skills across childhood.

However, there is evidence to suggest that there may be exceptions to this developmental pattern. Indeed, prior studies have also demonstrated that slight alterations in task conditions can enhance or attenuate children's performance (Enns \& Akhtar, 1989). Providing a central fixation point or location cuing in attention tasks (such as the flanker paradigm) allows children to attain performance levels similar to adults (Brodeur, 2004; Enns \& Akhtar, 1989). Preschool-aged children have also been shown to benefit from the addition of cues that highlight targets in filtering paradigms (Pastò \& Burack, 1997). Likewise, Pritchard and Neumann (2004) built on the work of Tipper and colleagues (Tipper, Bourque, Anderson, \& Brehaut, 1989; Tipper \& McLaren, 1990) in order to link specific underlying mechanisms to cognitive performance, and found that inhibitory processes associated with conceptual negative priming are intact in children as young as 5 years of age when the repetition of distractor and neutral conditions is omitted. However, the general paucity of research addressing this issue suggests that attempts to investigate mechanisms underlying attentional processes and cognitive control may have been hampered by difficulties with task design and experimental parameters.

Nonetheless, a growing number of paradigms have been designed specifically to measure cognitive skills during the preschool years. Rothbart and colleagues (Berger,
Jones, Rothbart, \& Posner, 2000; Jones, Rothbart, \& Posner, 2003; Posner \& Rothbart, 2000) have focused on the attentional and effortful control processes related to cognitive development through the use of both computer tasks (i.e., alert task, orienting task, spatial conflict task) and behavioral paradigms (i.e., Simple Simon task). This work has examined the interplay between attention shifting and focusing within a developmental perspective and also suggests that individual differences in effortful control over attention processes may be related to the growth of frontal neural circuitry that is prominently involved in error detection and conflict resolution. Zelazo and colleagues (Jacques \& Zelazo, 2001; Kerr \& Zelazo, 2004; Zelazo, Frye, \& Rapus, 1996; Zelazo, Reznick, \& Piñon, 1995) have also developed paradigms to investigate the development of regulatory processes and executive functioning (i.e., dimensional change card sort, DCCS, and flexible item selection task, FIST). These tasks tap into cognitive flexibility as well as children's ability to abstract rules and adapt appropriate behavioral responses.

The common goal of these paradigms is to gain greater insight into the cognitive processes related to the development of adaptive behavioral control in children. However, in order to determine the most appropriate experimental designs with which to examine cognitive development, we need to more accurately understand and predict the impact that variations in stimuli have on performance and strategy use in children of various ages. The present study attempts to examine the stability of selective attention skills and/or strategies across context by varying the characteristics of task stimuli in a computerized flanker paradigm. This paradigm has been utilized in numerous studies of selective attention in order to investigate a variety of related skills including susceptibility to interference, cuing effects, and spatial attention (e.g., Eriksen \& Eriksen, 1974; Green \& Bavelier, 2003; Huang-Pollock, Carr, \& Nigg, 2002; Ridderinkhof \& van der Molen, 1995; Ridderinkhof, van der Molen, Band, \& Bashore, 1997). By assessing the expression of cognitive skills (as measured by selective attention and associated regulatory processes) in the context of a computer paradigm, we hope to determine the suitability of these tasks for use with children between 4 and 6 years old. Future research can then employ specific task manipulations designed to examine the mechanisms underlying selective attention throughout development.

More specifically, this study hopes to ascertain which task versions are most appropriate for accurate betweenage-group comparisons. The flanker paradigm (Eriksen \& Eriksen, 1974) was chosen because of its use of nonlanguage-based stimuli, which require a motor rather than a verbal response, and the forced-choice nature of the task, which allows for simultaneous investigation of resistance to inference behavior and response control. Although previous studies with school-aged children have used versions of the flanker paradigm with arrows (Henderson, 2003; Ridderinkhof et al., 1997), geometric figures (Enns \& Akhtar, 1989), and fish (Rueda et al., 2004), the appropriateness of these various task designs in children of differing ages has yet to be fully examined. Thus, in the present study, three versions of the flanker paradigm 
were designed, which varied in hue (colors), dimensions (shapes), and direction (fish).

For each version, children were required to respond to a centrally located target despite the presence of interfering stimuli (see Figure 1 for stimuli examples). In trials in which flanking stimuli interference is low, subjects tend to have faster reaction times (RTs) than in trials with high levels of interference. This response pattern is referred to as interference (Eriksen \& Eriksen, 1974) or congruence effects (Ridderinkhof et al., 1997). In addition, two cognitive regulatory processes were also assessed: behavioral self-monitoring and response control. These processes are easily assessed in the flanker paradigm and are well suited for the investigation of individual differences in relation to cognitive development.

Differences in response latencies following erroneous and correct responses are thought to reflect behavior monitoring. Individuals who find an incorrect response more personally salient exhibit more controlled and slower responses after commission of an error (Davies, Segalowitz, \& Gavin, 2004; Henderson, 2003; Luu, Collins, \& Tucker, 2000). Response control reflects individual differences in overall performance strategy by assessing the degree of control or impulsivity evident in participants' RTs for correct and incorrect trials (Pailing, Segalowitz, Dywan, \& Davies, 2002). The key distinction between these measures is in the point of assessment. Behavior monitoring is a compensatory strategy evoked after an inaccurate response, in order to maximize future trial performance. In contrast, response control examines trial-by-trial performance strategy and is time-locked to the presence of correct and incorrect responses.

Determining the best computerized flanker version with which to assess the development of these two regulatory processes in children may help to inform our understanding of the transition from early reactive and automatic forms of regulation to later forms that are more controlled and voluntary. In particular, the transition to voluntary self-regulation has been speculated to underlie a wide range of dysregulated behaviors in children by increasing vulnerability to attention problems (i.e., attention-deficit hyperactivity disorder, ADHD), poor impulse control, aggression problems, and overactivation of fear circuits associated with anxiety and social withdrawal (Derryberry \& Rothbart, 1997).

In order to determine the functional associations between attentional processes, self-regulatory strategies, and behavioral outcomes, further research is needed to explore the multifaceted aspects of cognition within a developmental context (Bunge, Dudukovic, Thomason, Vaidya, \& Gabrieli, 2002). The present study attempts to examine young children's variations in cognitive skills in the form of interference effects and performance monitoring across three structurally similar, but visually distinct, versions of the flanker paradigm. It is hypothesized that typical response latency differences will be evident across the age groups and that performance will also vary by age with regard to interference effects and regulation. The youngest children should display greater susceptibility to interference and less self-monitoring.

\section{METHOD}

\section{Participants}

A total of 44 typically developing preschool- and kindergartenaged children $(M=5$ years, 3 months; range $=3.9-6.8 ; S D=.842$; 28 males, 16 females) participated in the study. These children represented three age groups, consisting of fourteen 4-year-olds $(M=$ 4 years, 5 months; range $=3.9-4.11 ; S D=.353$ ), eighteen 5 -yearolds $(M=5$ years, 5 months; range $=5.0-5.11 ; S D=.041)$, and twelve 6-year-olds $(M=6$ years, 3 months; range $=6.0-6.8 ; S D=$ $.029)$. Approximately half of the children $(n=25)$ were recruited from the University of Maryland's Center for Young Children; the rest of the sample $(n=19)$ was recruited from a list of participants obtained from an independent mailing company that provided addresses of families with young children located in the Washington, D.C. region.

\section{Apparatus}

Prior to the presentation of each flanker version, the children were asked to name pictures of the test stimuli (i.e., red and green circles, white stars and triangles, or purple fish) and point to the target stimulus that was in the "middle" of a row of five stimuli. These instructions were used to test for stimulus familiarity, impaired color vision, and comprehension of task terminology. None of the participants exhibited difficulty with these tasks. The children were then seated approximately $60 \mathrm{~cm}$ in front of a computer monitor and were asked to hold a small box $(5 \times 5 \times 1.5 \mathrm{in}$.). Two pushbuttons were located on the upper portion of the box; each button was .5 in. in diameter. Removable stickers that corresponded to the target stimuli were attached directly above the pushbuttons. Stickers were changed prior to each of the three versions of the flanker paradigm, which varied on hue, dimensionality, and direction.

\section{Procedure}

The flanker task assesses an individual's ability to inhibit predominant response tendencies in the face of interfering stimuli. Three computer versions of the flanker paradigm were developed, containing colors, shapes, and fish (see Figure 1). Each version was presented in discrete blocks of 32 trials for a total of 96 trials across all three versions. The stimuli were $1 \times 1 \mathrm{in}$. and were displayed in a horizontal row in the middle of a 17-in. monitor. Beneath the central target was a small circular fixation point approximately $0.5 \mathrm{~cm}$ in size.

Each version of the flanker paradigm was preceded by a practice round consisting of eight trials. The first block of stimuli consisted of red and green circles, the second block consisted of black and white shapes (triangles and stars), and the third block of stimuli consisted of fish that faced either right or left. Children were asked to respond to the central target on the screen, regardless of the nature of the flanking distractor items, by pressing the right button for one type of stimuli (i.e., red circle, star, or right-facing fish) and the left button for the opposite type of stimuli (i.e., green circle, triangle, or left-facing fish; see Figure 1 for stimulus examples). Congruent trials consisted of a target flanked by four identical stimuli, and incongruent trials had four flanking stimuli that were the opposite of the target. Equal numbers of congruent and incongruent trials were presented in a fixed random order within each flanker version.

Every trial began with a $300-\mathrm{msec}$ warning cue followed by an asterisk, a 500-msec fixation point, and the target display, which lasted for $700 \mathrm{msec}$. Both the 5- and 6-year-olds were required to respond within $1,300 \mathrm{msec}$ of the target presentation. The 4-yearolds were required to respond within $2,000 \mathrm{msec}$, in line with the findings of developmental response literature (Davies et al., 2004; Hale, 1990; Kail, 1986, 1993). Following response execution or lapse of the response window, feedback was presented on the screen as either a 1-in. smiley face (correct response) or a frowning face (incorrect response or no response). Feedback remained on the screen for $800 \mathrm{msec}$. For the 5- and 6-year-olds, the intertrial interval (ITI) varied randomly between 3,900 and 4,400 $\mathrm{msec}$. For the 4-year-olds, the ITI varied randomly between 5,900 and $6,400 \mathrm{msec}$. Stimulus 


\section{Congruent Stimuli Incongruent Stimuli}

Colors Version
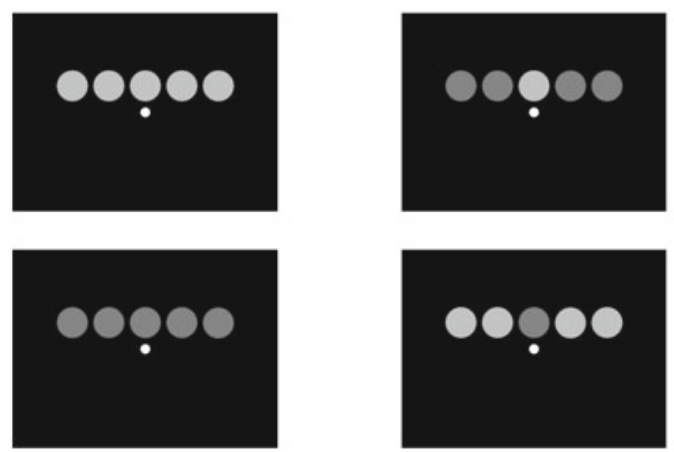

Shapes Version
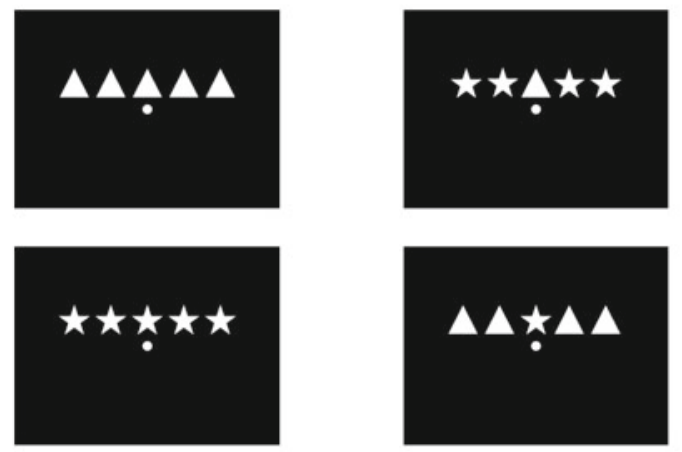

Fish Version
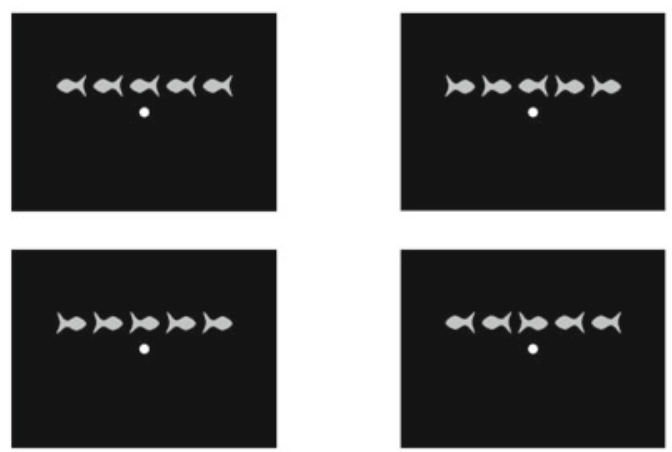

Figure 1: Flanker version stimuli.

presentation was controlled by computer software (STIM Stimulus Presentation Systems, James Long Company, Caroga Lake, NY) run on an IBM PC. Subject RT and response accuracy per trial were directly recorded by STIM program software. The entire task took approximately $25 \mathrm{~min}$ to complete.

\section{Statistical Analyses}

Data analyses were conducted with the Statistical Package for Social Sciences (SPSS) software, Version 12.0, produced by SPSS Incorporated. The analyses employed a series of repeated measures ANOVAs examining each of the behavioral outcomes of interest. Post hoc comparisons of significant interactions employed the Tukey test. $N \mathrm{~s}$ varied across analyses, because not all of the children were able to provide complete data in each flanker version; $N \mathrm{~s}$ are there- fore reported accordingly throughout the results section. In order to minimize the risk for Type I error, the Greenhouse-Geisser (G-G) procedure was applied when appropriate (Greenhouse \& Geisser, 1959). The degrees of freedom indicated in the test are those before the $\mathrm{G}-\mathrm{G}$ correction. However, epsilon $(\varepsilon)$ was noted when it was less than 1.0. RTs based on the data analyses are presented in Table 1 for the different trial types on each of the flanker versions across the three age groups.

Since the order of presentation for the flanker versions was consistent across all subjects, we examined the data for any signs of an order effect. Each version of the flanker task was subdivided into four sets of trials to examine subject performance throughout each individual task and also across the three tasks. Statistical analyses examining the interaction of these variables indicated no clear pattern of practice effects or order effects across all subjects $[F(6,216)=$ 1.10$, n.s. $]$, and within each age group $[F(12,204)=.94$, n.s. $]$.

\section{RESULTS}

\section{Attentional Processes}

A repeated measures ANOVA was used in order to examine interference effects in the task. Interference level (low for congruent trials or high for incongruent trials) and flanker version served as the within-subjects variables, age group was the between-subjects variable (4-year-olds, $n=$ 11; 5-year-olds, $n=17$; and 6-year-olds, $n=11$ ), and RT on trial type served as the dependent variable. All trials, both correct and incorrect, were included in this analysis. Subjects responded faster on congruent trials $(M=$ $824 \mathrm{msec})$ than on incongruent trials $(M=909 \mathrm{msec})$, producing a main effect for interference level $[F(1,36)=$ $55.55, p<.001$ ], indicating task-appropriate interference effects across all three versions of the flanker paradigm. In addition, there was a main effect for flanker version $[F(2,72)=7.63, p<.001]$, with significantly faster responses noted in the colors version $(M=823 \mathrm{msec})$ than in the fish $[M=883 \mathrm{msec} ; t(38)=-3.94, p<.001]$ and shapes $[M=.893 \mathrm{msec} ; t(38)=2.35, p<.02]$ versions. There was also a main effect for age group $[F(2,36)=$ $30.05, p<.001$ ], with the 4-year-olds responding significantly slower overall $(p \mathrm{~s}<.001)$. This was qualified by an interaction between flanker version and age group $[F(4,72)=2.38, p<.059]$. The 4-year-olds exhibited slower RTs on both the shapes and fish versions compared with the colors version $[t(10)=2.19, p<.05$; $t(10)=3.41, p<.01$, respectively]. In contrast, both the 5- and 6-year-olds displayed significantly slower RTs only when comparing the shapes version with the colors version $[t(16)=3.09, p<.01 ; t(10)=2.19, p<.05$, respectively].

Percentage of errors in response execution was examined with a repeated measures ANOVA using flanker type and congruency (congruent or incongruent trials) as the within-subjects factors and age group as the betweensubjects factor (4-year-olds, $n=11$; 5-year-olds, $n=16$; and 6-year-olds, $n=11$ ). A main effect was found for congruency $[F(1,35)=87.12, p<.001]$, with a higher percentage of errors occurring on incongruent $(M=16.1 \%)$ as opposed to congruent $(M=10.2 \%)$ trials. There was also a main effect for version $[F(2,70)=5.30, p<.01$, $\varepsilon=.814]$ but not for age group $[F(2,35)=.02$, n.s. $]$. 
Table 1

Reaction Times (RTs, in Milliseconds) for Trial Type and Flanker Version

\begin{tabular}{|c|c|c|c|c|c|c|c|c|c|c|c|c|c|}
\hline \multirow{3}{*}{$\begin{array}{l}\text { Flanker } \\
\text { Version }\end{array}$} & \multirow{3}{*}{$\begin{array}{c}\text { Age } \\
\text { (Years) }\end{array}$} & \multicolumn{8}{|c|}{ Trial Type } & \multirow{2}{*}{\multicolumn{2}{|c|}{$\begin{array}{c}\text { After } \\
\text { Correct } \\
\text { Trials }\end{array}$}} & \multirow{2}{*}{\multicolumn{2}{|c|}{$\begin{array}{c}\text { After } \\
\text { Incorrect } \\
\text { Trials }\end{array}$}} \\
\hline & & \multicolumn{2}{|c|}{ Congruent } & \multicolumn{2}{|c|}{ Incongruent } & \multicolumn{2}{|c|}{ Correct } & \multicolumn{2}{|c|}{ Incorrect } & & & & \\
\hline & & $\mathrm{RT}$ & $S D$ & RT & $S D$ & RT & $S D$ & RT & $S D$ & RT & $S D$ & $\mathrm{RT}$ & $S D$ \\
\hline Colors & $\begin{array}{l}4 \\
5 \\
6\end{array}$ & $\begin{array}{l}934 \\
692 \\
734\end{array}$ & $\begin{array}{l}.15 \\
.13 \\
.16\end{array}$ & $\begin{array}{r}1,066 \\
737 \\
774\end{array}$ & $\begin{array}{l}.19 \\
.13 \\
.12\end{array}$ & $\begin{array}{l}983 \\
711 \\
752\end{array}$ & $\begin{array}{l}.14 \\
.11 \\
.11\end{array}$ & $\begin{array}{l}815 \\
669 \\
622\end{array}$ & $\begin{array}{l}.31 \\
.13 \\
.13\end{array}$ & $\begin{array}{l}941 \\
687 \\
687\end{array}$ & $\begin{array}{l}.20 \\
.11 \\
.10\end{array}$ & $\begin{array}{l}990 \\
722 \\
725\end{array}$ & $\begin{array}{l}.17 \\
.13 \\
.13\end{array}$ \\
\hline Shapes & $\begin{array}{l}4 \\
5 \\
6\end{array}$ & $\begin{array}{r}1,053 \\
754 \\
761\end{array}$ & $\begin{array}{l}.23 \\
.11 \\
.12\end{array}$ & $\begin{array}{r}1,158 \\
805 \\
828\end{array}$ & $\begin{array}{l}.19 \\
.11 \\
.14\end{array}$ & $\begin{array}{r}1,057 \\
784 \\
794\end{array}$ & $\begin{array}{l}.19 \\
.10 \\
.12\end{array}$ & $\begin{array}{l}973 \\
687 \\
667\end{array}$ & $\begin{array}{l}.27 \\
.10 \\
.18\end{array}$ & $\begin{array}{r}1,046 \\
748 \\
763\end{array}$ & $\begin{array}{l}.24 \\
.08 \\
.13\end{array}$ & $\begin{array}{r}1,123 \\
813 \\
792\end{array}$ & $\begin{array}{l}.17 \\
.14 \\
.13\end{array}$ \\
\hline Fish & $\begin{array}{l}4 \\
5 \\
6\end{array}$ & $\begin{array}{r}1,055 \\
704 \\
730\end{array}$ & $\begin{array}{l}.15 \\
.13 \\
.15\end{array}$ & $\begin{array}{r}1,238 \\
769 \\
803\end{array}$ & $\begin{array}{l}.19 \\
.14 \\
.13\end{array}$ & $\begin{array}{r}1,104 \\
731 \\
727\end{array}$ & $\begin{array}{l}.17 \\
.13 \\
.11\end{array}$ & $\begin{array}{r}1,072 \\
690 \\
695\end{array}$ & $\begin{array}{l}.14 \\
.13 \\
.19\end{array}$ & $\begin{array}{r}1,089 \\
701 \\
707\end{array}$ & $\begin{array}{l}.18 \\
.11 \\
.11\end{array}$ & $\begin{array}{r}1,139 \\
745 \\
713\end{array}$ & $\begin{array}{l}.21 \\
.14 \\
.19\end{array}$ \\
\hline
\end{tabular}

Post hoc analyses on the version main effect indicated that the fish version elicited more errors than the colors $[t(37)=2.43, p<.02]$ and shapes $[t(37)=3.02, p<$ $.01]$ versions. Furthermore, there was also a significant interaction between congruency and version $[F(2,70)=$ $10.09, p<.001]$. Pairwise $t$ tests revealed that the difference between percentage of errors on congruent as compared with incongruent trials was significantly greater for the fish version than for the colors $[t(37)=3.24, p<$ $.001]$ and shapes $[t(37)=3.89, p<.003]$ versions. In accordance with the interference effects results, in which children had the fastest RTs in the colors version, children also exhibited the fewest errors in the colors version. Likewise, when examining RTs and errors on congruent as compared with incongruent trials, children responded faster on congruent trials, while committing more errors on incongruent trials.

To further examine the relationship between trial type and response accuracy, RT data on correct versus incorrect trials were examined with a repeated measures ANOVA. Accuracy (correct or incorrect trials) and flanker version were the within-subjects factors; age group served as the between-subjects variable (4-year-olds, $n=9$; 5-yearolds, $n=15$; and 6-year-olds, $n=8)$. Main effects were found for accuracy $[F(1,29)=26.44, p<.001]$, version $[F(2,58)=4.55, p<.02]$, and age group $[F(2,29)=$ $23.70, p<.001]$. Children responded faster on incorrect trials $(M=765 \mathrm{msec})$ than on correct trials $(M=$ $849 \mathrm{msec}$ ). They also responded most quickly in the colors version, as opposed to the shapes $[t(31)=2.04, p<$ $.05]$ and fish $[t(31)=2.16, p<.04]$ versions. Again, the 4-year-olds had the slowest RTs across the tasks ( $p$ s $<$ $.001)$. A nonsignificant trend was also found for the interaction of flanker version and accuracy $[F(2,58)=2.91$, $p<.062]$. Pairwise $t$ tests indicated that the RT difference between correct and incorrect trials was significantly smaller in the fish version than in the shapes version $[t(31)=2.25, p<.03]$. Overall, the accuracy data indicate that errors are associated with faster RTs. These results are consistent with the notion that faster responding is a phenomenon associated both with mastery of a task (as in the case of congruent trials in comparison with incongruent trials) and impulsive response execution, in which lack of appropriate processing time translates into a faster average RT on incorrect trials. These notions, although seemingly contradictory in nature, are commonly noted in the flanker paradigm literature (Pailing et al., 2002).

\section{Regulatory Skills}

To examine self-monitoring, a repeated measures ANOVA was run with monitoring (RT after correct or RT after incorrect trials) as the within-subjects variable; age group served as the between-subjects factor (4-year-olds, $n=9$; 5-year-olds, $n=14$; and 6-year-olds, $n=7$ ). RT on the trial following a correct or incorrect trial was the dependent variable. Main effects were evident for both monitoring $[F(1,27)=5.93, p<.02]$ and flanker version $[F(2,54)=6.23, p<.001]$. Subjects exhibited faster RTs on trials following correct responses $(M=819 \mathrm{msec})$ than on trials following incorrect responses $(M=863 \mathrm{msec})$. This finding is in line with previous results from both children and adults that indicate a general tendency to maximize response accuracy by slowing RT after an error (Davies et al., 2004; Henderson, 2003; Pailing et al., 2002). For the version main effect, subjects had a significantly slower overall RT on the shapes version $[t(29)=3.85$, $p<.001]$ than on the colors version of the flanker paradigm. No main effect was found for age group, and there were also no significant interactions.

Interestingly, there were individual children within the sample who did not demonstrate self-monitoring within each of the flanker types. Therefore, two groups of subjects, monitors and nonmonitors, were established for each flanker version using a simple dichotomous split based on average RT difference between trials after a correct response and trials after an error. Children who had slower average RTs on trials following an error versus trials following correct responses were categorized as selfmonitors. Children who exhibited the opposite pattern (faster average RTs after the commission of an error) were categorized as non-self-monitors. In the present study, the majority of children exhibited self-monitoring. This pattern was evident in the colors (25-13), shapes (26-13), and fish (23-11) tasks, with no statistical differences between the number of monitors and nonmonitors for any of the three versions $\left(\chi^{2} \mathrm{~s}<2.64, p s>.60\right)$. Further examination of these groups also indicated no significant 
difference in the total number of monitoring versus nonmonitoring subjects for each age group of children across all three flanker versions $\left(\chi^{2} \mathrm{~s}<.70, p \mathrm{~s}>.42\right)$. This result is consistent with previous work involving children and adults, which suggests that factors other than a child's age, such as inhibitory control and temperamental traits, may influence the expression of self-monitoring and regulatory behaviors (Henderson, 2003; Pailing \& Segalowitz, 2004).

Following the statistical strategy used by Pailing and colleagues (2002), differences in response speed can be accounted for with RT residual scores. These are created by regressing correct trial RTs onto incorrect trial RTs. Positive residual scores (small RT differences between correct and incorrect trials) signify a controlled, or cautious, style of responding, whereas more negative residual scores (large RT differences between correct and incorrect trials) indicate an impulsive response approach. Subjects were then median split into two groups-low response control and high response control. An independent samples $t$ test examining overall RT on correct trials for each flanker version demonstrated that the low-response control subjects were not faster responders in general $(t \mathrm{~s}<$ $.49, p \mathrm{~s}>.63$, n.s.). Thus, response control differences should theoretically be indicative of error rates, with the low-response control group exhibiting a higher susceptibility to the execution of errors.

However, there were no significant group differences in the total number of errors for any of the three flanker versions $(t \mathrm{~s}<.11, p \mathrm{~s}>.91, \mathrm{n} . \mathrm{s}$. $)$, nor was there a significant correlation between total number of errors and residual RT difference scores. There was also no relation between subject age and response control for any of the flanker versions $(r \mathrm{~s}<.18, p \mathrm{~s}>.29$, n.s. $)$. These results are dissimilar to data compiled with adult subjects (Pailing et al., 2002), and the discrepancy suggests that response control capacities in children may not represent the same level of skill, or strategy use, as found in adult performance on similar cognitive tasks.

An additional set of analyses was run to examine the relationship between self-monitoring and response control. Subjects who exhibited self-monitoring had a significantly more positive response control score $[t(32)=2.26, p<$ $.03]$ in the fish version of the task. This relation between self-monitoring and increased response control was present, however, in the colors version, and there was essentially no monitoring group difference in response control RTs for the shapes version of the flanker paradigm.

\section{DISCUSSION}

This study was designed to examine the influence of task variation on selective attention and regulatory control. Three versions of the flanker paradigm were used to compare, across age groups, potential changes in response speed, interference effects, response accuracy, and self-monitoring. As anticipated, all three versions of the flanker paradigm tested in this study elicited the typical interference effects associated with the flanker paradigm, such that RTs on congruent trials were faster than RTs on incongruent trials. However, the interaction between congruency and flanker version seems to suggest that there are not equivalent levels of processing occurring across the three versions. Although the 6-year-olds did not exhibit significant RT differences between congruent and incongruent trials on any version, the 5-year-olds needed more time to respond to stimuli in the incongruent trials for the shapes version, whereas the 4-year-olds took longer on incongruent trials in both the shapes and fish versions. These results suggest at least three possibilities: (1) The 6-year-olds may be employing additional performance strategies to equalize response patterns across all versions, (2) the perceptual demands of the stimuli are less taxing to the older children's cognitive capacity, or (3) the older children do not experience as large a "bottleneck" from the simultaneous competing responses seen in the incongruent trials. To examine these possibilities more closely, we turn to the additional data analyses.

The children responded more quickly to the incorrect trials than to the correct trials. This pattern is commonly attributed to a speed-accuracy trade-off, whereby impulsive (quick) responses are more likely to result in an error. In this study, the RT difference between correct and incorrect trials may have been influenced by the children's greater familiarity with particular aspects of the task components and may also reflect the use of particular performance strategies. Since the smallest RT difference was found in the fish version, it is possible that children were employing a knowledge rule based on directionality in their responding. Specifically, rather than creating a new response mapping between random stimuli and the right or left hand, performance on the fish version may have been easier for those children who already knew right from left and were able to apply this knowledge to the task. For example, children of this age have some understanding of directionality, and several subjects expressed comprehension of "right" versus "left" during the fish version of the task. Thus, perceptual processing may be equally difficult across version - however, familiarity with direction could have been a strategy employed by some of the children, particularly in the older age groups. The methodology employed in testing the children refrained from introducing the terminology "left" and "right," and unfortunately there was no debriefing question to ascertain whether or not this was a skill used by the children.

Initially, the fish stimuli were anticipated to generate the most interest and focus in the children, while simultaneously providing the most difficulty in terms of attentional allocation. However, the present results indicate that prior task knowledge (i.e., greater familiarity with the concepts of right and left) may have had an impact on the children's performance. These data are in line with research on elementary-school-aged children, demonstrating that older children are more skilled at understanding the relevant aspects of task dimensions and thereby more efficient in their attentional focus and task performance than younger children (Barrett \& Shepp, 1988; Lane \& Pearson, 1982; Pick, Christy, \& Frankel, 1972). Thus, it is possible that perceptual demands activated by various stimuli can interact with attention skills, such as filtering 
and resistance to interference, depending on a subject's age and prior knowledge.

Nonetheless, when discussing perceptual skills such as filtering, it is important to note that the flanker paradigm used in this study was designed to keep attentional competition limited to local rather than global processing. Subjects were required to examine the individual aspects of each stimulus on the screen rather than grouping the set of stimuli as a whole object. Children's local processing is believed to come online prior to global processes, and global processing has also been shown to be more affected by variations in stimuli structure and task complexity (Burack, Enns, Iarocci, \& Randolph, 2000; Dukette \& Stiles, 2001). Thus, it is less likely that perceptual skills were being overtaxed in this study. Rather, strategy use or conflict-resolution issues are more likely underlying the differences in performance.

Across all versions, a higher percentage of errors occurred on incongruent than on congruent trials, again demonstrating that these versions of the flanker paradigm were producing typical interference effects. The least errors occurred in the colors versions, followed by the shapes and then the fish versions of the task. This pattern was also found in the interaction between congruency and flanker version, with children exhibiting the greatest difference in error percentage between congruent and incongruent trials in the fish version in comparison with both the colors and shapes versions. This trend is consistent with the notion that the strategic use of directionality knowledge created stronger response competition during incongruent trials on the fish version. Thus, children's attention skills are being taxed more strongly by the incompatible stimuli that automatically and simultaneously activate an alternative response due to knowledge of directionality. This may be the case for the present results on the fish version of the task. Interestingly, no age group differences were found for percentage of errors. This may be due to the nature of the sample used in this study. The children primarily came from middle- to upper-class families in which the majority of children may have developed a good sense of directionality at a young age.

Overall, the combination of interference effects and RT patterns on correct and incorrect trials is in line with previous flanker task findings with adults and older children (Davies et al., 2004; Henderson, 2003; Pailing et al., 2002), indicating that all versions of the task were producing the standard flanker effect. However, the variation in RT and response accuracy between the different flanker versions indicates that differing task components may result in more or less satisfactory levels of task difficulty. Awareness of these subtle, yet consequential, differences allows for more appropriate use of computerized research paradigms.

This concern is also pertinent within the context of developmental considerations. Research paradigms are commonly applied across a wide age range of subjects, and comparison of child, adolescent, and adult outcomes can occur without prior verification that each age group has employed comparable strategies or that similar cognitive processes have been activated across the various age groups. In addition, atypical populations are often com- pared, and although there may be a smaller age range of children, these children are often more developmentally diverse, with a fair amount of variation in cognitive skills. Consequently, when examining populations that may have a wide range of skills, it is important to use the most basic version of a paradigm in order to avoid attributing discrepancies in attention performance to developmental differences in perceptual skills or attentional mechanism, when the observed behavior may be due to additional strategy use linked to the task parameters. For instance, in the Stroop task, children must be proficient readers in order to elicit the stimulus-response conflict at the core of the classic Stroop effect (Besner \& Stolz, 1999).

In this experiment, the colors version appeared to be the simplest task; children across all age groups exhibited the greatest ease in responding quickly and accurately while also displaying the typical interference effects associated with the flanker paradigm. The shapes version would also be appropriate to use with a wide age range of children, because the variation in shape dimension corresponds to the mappings of the stimuli in letter variations of the flanker paradigm. Lastly, the fish version would be best suited for populations of older children (elementary school aged), since there is greater variation in young children's exposure to preschool environments, in which information such as directionality would typically be taught. Further research is necessary on the variation of experimental contexts in order to determine the magnitude of interference effects when manipulating the forms of distracting stimuli on the incongruent trials. For example, the present study employed distractors that only indicated the opposite response of the target item; future studies should examine the potential impact of neutral distractors and/or the number of distractors on preschoolers' cognitive performance.

For investigations primarily concerned with the inhibitory mechanisms involved with selective attention processes, the basic versions of colors or shapes would serve well. The fish version could also be used. However, care would need to be taken to determine that all subjects had equivalent knowledge of information pertinent to potential strategy use (i.e., directionality). Recent work by Pritchard and Neumann (2004) also supports the suggestion that tasks using color and shape stimuli are appropriate when designing tasks tapping the mechanisms underlying attentional performance.

The present study also examined the prevalence of self-monitoring in preschool- and kindergarten-aged children. The data indicate that self-monitoring was prevalent across all flanker versions. However, some participants actually demonstrated faster responding rather than a slowing of RT after an error. Since this pattern was apparent for individual subjects in all versions, the results suggest that factors in addition to age level may play a role in the development and expression of self-monitoring strategies. For instance, prior research in older children and adults indicates that personality variables, including temperament and negative affect, may contribute to variations in self-monitoring (Davies et al., 2004; Henderson, 2003; Luu et al., 2000). 
Furthermore, these personality variables may also be related to the patterns of response control found in this sample of children. We anticipated that impulsive responding would be related to age (in months), with the younger children being more likely to display impulsive responding. On the contrary, the data indicate that response control was not dependent on subject age or task performance. We did find a relation between self-monitoring strategy and response control. In fact, the pattern of results is suggestive of several alternative hypotheses: (1) attentional processes (i.e., susceptibility to interference) and associated task performance strategies may have divergent developmental time frames, (2) there may be a potential interaction between task difficulty (as assessed by the different versions of the computerized task) and children's attentional skill level, or (3) performance strategies may be more dependent on individual differences (i.e., temperament or personality factors) due to their influence on children's attentional skills, motivation levels, and behavioral patterns. Together, the results of the self-monitoring and response control tests present a somewhat complicated picture of emerging, but perhaps not purely refined, regulatory strategies that may be influenced by task components and individual differences in task strategy.

Although personality and temperament traits were not investigated in the present study, future research should account for these factors. Future studies should also move beyond purely behavioral measures. However, since behavioral data did not yet exist on the newly designed versions of the flanker paradigm, it was not advantageous at the time of this study to collect physiological data. A future project will examine the correlation between behavioral and biological assessments of self-monitoring within the context of these task versions. The combination of behavioral and physiological methodology would contribute significantly to our understanding of the developmental progression of self-monitoring and response control. Lastly, another expansion to the present study would involve investigating the influence of training on young children's ability to perform successfully on tasks in which stimuli may be creating an additional perceptual burden that interferes with the speed and/or efficiency of cognitive processing. Specifically, the influence of task training as related to the presentation sequence of various forms of stimuli should be examined in future studies, and counterbalancing the three versions would help control for potential practice effects.

\section{Conclusions}

During early childhood, children experience a dramatic improvement in their ability to utilize attentional skills under increasingly complex conditions. This allows children to flexibly adapt their behavior to suit specific task demands. The major benefits of the present study include a more detailed understanding of the influence of task component variation on selective attention skills (i.e., interference effects) and the emergence of performance strategies (i.e., self-monitoring and response control) in early childhood. Surprisingly, not all children who were anticipated to exhibit self-monitoring, on the basis of their age, exhibited this specific response strategy. Therefore, these data suggest a need to investigate additional factors besides numerical age that may be related to the development of cognitive skills. Overall, this study demonstrated that the three computerized versions of the flanker task were differentially sensitive to examining children's attentional skills and performance outcome strategies. Additional work is needed to clarify these discrepancies within a broader developmental context. A focus on the task manipulations would allow for more detailed investigation of the mechanisms underlying the cognitive processes tapped in the flanker paradigm.

\section{AUTHOR NOTE}

The project was supported by The Early Experience, Stress Neurobiology, and Prevention Science Network, directed by Megan Gunnar (NIMH Grant 1 R21 MH65046). J.M.M. is a trainee of this network. This project was also partially supported by NIH Grant HD17899, awarded to N.A.F. K.P.-E. is now at George Mason University. Correspondence concerning this article should be addressed to J. M. McDermott, Department of Human Development, University of Maryland, 3304 Benjamin Building, College Park, MD 20742 (e-mail: jnmartin@umd.edu).

\section{REFERENCES}

Barrett, S. E., \& Shepp, B. E. (1988). Developmental changes in attentional skills: The effect of irrelevant variations on encoding and response selection. Journal of Experimental Child Psychology, 45, 382-399.

Berger, A., Jones, L., Rothbart, M. K., \& Posner, M. I. (2000). Computerized games to study the development of attention in childhood. Behavior Research Methods, Instruments, \& Computers, 32 297-303.

BeSNer, D., \& Stolz, J. A. (1999). Unconsciously controlled processing: The Stroop effect reconsidered. Psychonomic Bulletin \& Review, 6, 449-455.

BJoRKLUnd, D. F., \& HARNishfeger, K. K. (1990). The resources construct in cognitive development: Diverse sources of evidence and a theory of inefficient inhibition. Developmental Review, 10, 48-71.

Brodeur, D. A. (2004). Age changes in attention control: Assessing the role of stimulus contingencies. Cognitive Development, 19, 241-252.

Bunge, S. A., Dudukovic, N. M., Thomason, M. E., Vaidya, C. J., \& GABRIELI, J. D. E. (2002). Immature frontal lobe contributions to cognitive control in children: Evidence from fMRI. Neuron, $\mathbf{3 3}$ 301-311.

Burack, J. A., \& Enns, J. T. (1997). Attention, development, and psychopathology. New York: Guilford.

Burack, J. A., Enns, J. T., Iarocci, G., \& Randolph, B. (2000). Age differences in visual search for compound patterns: Long- versus short-range grouping. Developmental Psychology, 36, 731-740.

Carver, A. C., Livesey, D. J., \& Charles, M. (2001). Age related changes in inhibitory control as measured by stop signal task performance. International Journal of Neuroscience, 107, 43-61.

CASE, R. (1985). Intellectual development: Birth to adulthood. New York: Academic Press.

Casey, B. J., Tottenham, N., \& Fossella, J. (2002). Clinical, imaging, lesion, and genetic approaches toward a model of cognitive control. Developmental Psychobiology, 40, 237-254.

Davies, P. L., Segalowitz, S. J., \& Gavin, W. J. (2004). Development of response-monitoring ERPs in 7- to 25-year-olds. Developmental Neuropsychology, 25, 355-376.

Dempster, F. N. (1993). Resistance to interference: Developmental changes in a basic processing mechanism. In M. L. Howe \& R. Pasnak (Eds.), Emerging themes in cognitive development: Vol. 1. Foundations (pp. 3-27). New York: Springer.

DERrYberRy, D., \& Rothbart, M. K. (1997). Reactive and effortful processes in the organization of temperament. Development \& Psychopathology, 9, 633-652.

Desimone, R., \& Duncan, J. (1995). Neural mechanisms of selective visual attention. Annual Reviews in Neuroscience, 18, 193-222. 
DiAmOND, A. (1990). The development and neural bases of memory functions as indexed by the $\mathrm{AB}$ and delayed response tasks in human infants and infant monkeys. In A. Diamond (Ed.), The development and neural bases of higher cognitive functions (Annals of the New York Academy of Sciences, Vol. 608, pp. 267-317). New York: New York Academy of Sciences.

Dukette, D., \& Stiles, J. (2001). The effects of stimulus density on children's analysis of hierarchical patterns. Developmental Science, 4, 233-251.

ENNS, J. T., \& AKHTAR, N. (1989). A developmental study of filtering in visual attention. Child Development, 60, 1188-1199.

ENNS, J. T., \& CAMERon, S. (1987). Selective attention in young children: The relations between visual search, filtering, and priming. Journal of Experimental Child Psychology, 44, 38-63.

EnNs, J. T., \& Girgus, J. S. (1985). Developmental changes in selective and integrative visual attention. Journal of Experimental Child Psychology, 40, 319-337.

Eriksen, B. A., \& EriKsen, C. W. (1974). Effects of noise letters upon the identification of a target letter in a nonsearch task. Perception \& Psychophysics, 16, 143-149.

Green, C. S., \& Bavelier, D. (2003). Action video game modifies visual selective attention. Nature, 423, 534-537.

Greenhouse, S. W., \& Geisser, S. (1959). On methods in the analysis of profile data. Psychometrika, 24, 95-112.

Hale, S. (1990). A global developmental trend in cognitive processing speed. Child Development, 61, 653-663.

Halford, G. S. (1993). Children's understanding: The development of mental models. Hillsdale, NJ: Erlbaum.

HARNishfeger, K. K. (1995). The development of cognitive inhibition: Theories, definitions, and research evidence. In F. N. Dempster \& C. J. Brainerd (Eds.), Interference and inhibition in cognition (pp. 175 204). San Diego: Academic Press.

Henderson, H. A. (2003). Temperamental contributions to problem solving: Cognitive and affective processes. Dissertation Abstracts International, 64, 1B. (UMI No. 0419-4217)

Huang-Pollock, C. L., Carr, T. H., \& Nigg, J. T. (2002). Development of selective attention: Perceptual load influences early versus late attentional selection in children and adults. Developmental Psychology, 38, 363-375.

JaCQues, S., \& Zelazo, P. D. (2001). The Flexible Item Selection Task (FIST): A measure of executive function in preschoolers. Developmental Neuropsychology, 20, 573-591.

Jones, L. B., Rothbart, M. K., \& Posner, M. I. (2003). Development of executive attention in preschool children. Developmental Science, 6, 498-504.

KAIL, R. (1986). Sources of age differences in speed of processing. Child Development, 57, 969-987.

KAIL, R. (1993). Processing time decreases globally at an exponential rate during childhood and adolescence. Journal of Experimental Child Psychology, 56, 254-265.

KerR, A., \& ZelaZo, P. D. (2004). Development of "hot" executive function: The children's gambling task. Brain \& Cognition, 55, 148-157.

Lane, D. M., \& Pearson, D. A. (1982). The development of selective attention. Merrill-Palmer Quarterly, 28, 317-337.

Luu, P., Collins, P., \& Tucker, D. M. (2000). Mood, personality, and self-monitoring: Negative affect and emotionality in relation to frontal lobe mechanisms of error monitoring. Journal of Experimental Psychology: General, 129, 43-60.

Müller, U., Zelazo, P. D., Hood, S., Leone, T., \& Rohrer, L. (2004). Interference control in a new rule use task: Age-related changes, labeling, and attention. Child Development, 75, 1594-1609.

Pailing, P. E., \& Segalowitz, S. J. (2004). The error-related negativity as a state and trait measure: Motivation, personality, and ERPs in response to errors. Psychophysiology, 41, 84-95.

Pailing, P. E., Segalowitz, S. J., Dywan, J., \& Davies, P. L. (2002). Error negativity and response control. Psychophysiology, 39, 198-206.

Pastò, L., \& Burack, J. A. (1997). A developmental study of visual attention: Issues of filtering efficiency and focus. Cognitive Development, 12, 427-439.

Pick, A. D., Christy, M. D., \& Frankel, G. W. (1972). A developmental study of visual selective attention. Journal of Experimental Child Psychology, 14, 165-175.

Posner, M. I., \& Rothbart, M. K. (2000). Developing mechanisms of self-regulation. Development \& Psychopathology, 12, 427-441.

Pritchard, V. E., \& Neumann, E. (2004). Negative priming effects in children engaged in nonspatial tasks: Evidence for early development of an intact inhibitory mechanism. Developmental Psychology, 40, 191-203.

RidderinkhoF, K. R., \& van der Molen, M. W. (1995). A psychophysiological analysis of developmental differences in the ability to resist interference. Child Development, 66, 1040-1056.

Ridderinkhof, K. R., van der Molen, M. W., Band, G. P. H., \& BAsHore, T. R. (1997). Sources of interference from irrelevant information: A developmental study. Journal of Experimental Child Psychology, 65, 315-341.

Rueda, M. R., Fan, J., McCandliss, B. D., Halparin, J. D., Gruber, D. B., Lercari, L. P., \& Posner, M. I. (2004). Development of attentional networks in childhood. Neuropsychologia, 42, 1029-1040.

Shallice, T. (1988). From neuropsychology to mental structure. New York: Cambridge University Press.

Tipper, S. P., Bourque, T. A., Anderson, S. H., \& Brehaut, J. C. (1989). Mechanisms of attention: A developmental study. Journal of Experimental Child Psychology, 48, 353-378.

Tipper, S. P., \& McLaren, J. (1990). Evidence for efficient visual selectivity in children. In J. T. Enns (Ed.), The development of attention: Research and theory (pp. 197-210). Amsterdam: Elsevier.

VAN DER Molen, M. W. (2000). Developmental changes in inhibitory processing: Evidence from psychophysiological measures. Biological Psychology, 54, 207-239.

Wright, I., Waterman, M., Prescott, H., \& Murdoch-Eaton, D. (2003). A new Stroop-like measure of inhibitory function development: Typical developmental trends. Journal of Child Psychology \& Psychiatry, 44, 561-575.

Zelazo, P. D., Frye, D., \& Rapus, T. (1996). An age-related dissociation between knowing rules and using them. Cognitive Development, 11, 37-63.

Zelazo, P. D., Reznick, J. S., \& Piñon, D. E. (1995). Response control and the execution of verbal rules. Developmental Psychology, 31, 508-517.

(Manuscript received March 11, 2005; revision accepted for publication October 4, 2005.) 